\title{
Human Visual System-aware Dimming Method Combining Pixel Compensation and Histogram Specification for TFT-LCDs
}

\author{
Jeong-Chan Jin and Young-Jin Kim \\ Department of Electrical and Computer Engineering, Ajou University \\ San 5, Woncheon-dong, Yeongtong-gu, Suwon 443-749, Republic of Korea \\ [e-mail: akswolove@ajou.ac.kr, youngkim@ajou.ac.kr] \\ *Corresponding author: Young-Jin Kim
}

Received November 18, 2016; revised March 16, 2017; accepted August 9, 2017;

published December 31, 2017

\begin{abstract}
In thin-film transistor liquid-crystal displays (TFT-LCDs), which are most commonly used in mobile devices, the backlight accounts for about $70 \%$ of the power consumption. Therefore, most low-power-related studies focus on realizing power savings through backlight dimming. Image compensation is performed to mitigate the visual distortion caused by the backlight dimming. Therefore, popular techniques include pixel compensation for brightness recovery and contrast enhancement, such as histogram equalization. However, existing pixel compensation techniques often have limitations with respect to blur owing to the pixel saturation phenomenon, or because contrast enhancement cannot adequately satisfy the human visual system (HVS). To overcome these, in this study, we propose a novel dimming technique to achieve both power saving and HVS-awareness by combining the pixel compensation and histogram specifications, which convert the original cumulative density function (CDF) by designing and using the desired CDF of an image. Because the process of obtaining the desired CDF is customized to consider image characteristics, histogram specification is found to achieve better HVS-awareness than histogram equalization. For the experiments, we employ the LIVE image database, and we use the structural similarity (SSIM) index to measure the degree of visual satisfaction. The experimental results show that the proposed technique achieves up to $15.9 \%$ increase in the SSIM index compared with existing dimming techniques that use pixel compensation and histogram equalization in the case of the same low-power ratio. Further, the results indicate that it achieves improved HVS-awareness and increased power saving concurrently compared with previous techniques.
\end{abstract}

Keywords: TFT-LCDs, human visual system, low power, backlight dimming, histogram specification, pixel compensation, cumulative density function.

This work was supported by the National Research Foundation of Korea (NRF) grant funded by the Korean government (MSIP) (No. 2015R1A2A2A01008434). This work was also supported by the Ajou University research fund. 


\section{Introduction}

The display on mobile devices such as smartphones is a major means by which end users obtain information, and these displays are responsible for a significant amount of power consumption.

In recent years, thin film transistor liquid crystal displays (TFT-LCDs) have been widely used in mobile devices, including Apple and LG smartphones, and their backlight consumes up to $70 \%$ of the total power [1]. Thus, backlight dimming is essential for power saving in TFT-LCDs. However, because a reduction in display brightness results in visual distortion, image compensation is required to restore visual satisfaction.

Pixel compensation is a widely used technique to compensate for visual distortion because its calculation is simple, and it is highly effective in image recovery. For example, Kang et al. [2] and Chen et al. [3] attempted to combine pixel compensation with the structural similarity (SSIM) index [4], which is an image quality assessment (IQA) metric that reflects the human visual system (HVS). They calculated a target error based on the SSIM index and limited backlight dimming so that the target error in the image quality following pixel compensation is not exceeded. Kang et al. [5] proposed a segmentation-based clipped error control algorithm (SBCRCA) [6], and used it to conduct image compensation using backlight dimming methods based on IQA tools, suppressing the image distortion. Pixel compensation is often used for local dimming with different dimming scales at each of the image regions. Yang et al. [7] performed local dimming in an edge-lit backlight display and attempted nonuniform pixel compensation accordingly. However, the more the pixels are compensated, the greater is the saturation that is likely to occur. This result tends to make the image blurred. To improve this, Shen et al. [8] attempted visual enhancement using image decomposition, but it is far from HVS-awareness. Thus, because pixel compensation-based techniques often fail to dim in a sophisticated manner, research has focused on contrast enhancement rather than brightness restoration.

Because the execution of backlight dimming causes a decrease in both brightness and contrast [9] to some degree, there is extensive research into contrast compensation. One of the most widely used techniques for contrast enhancement is histogram equalization (HE). This method aims to make the histogram of the pixel values of an image uniform, and is effective in enhancing the visuality of low-contrast images [10]. Thus, HE is useful in compensating for contrast reduction that is due to display dimming. In order to apply this characteristic, Iranli et al. proposed histogram equalization for backlight scaling (HEBS) [11], which combines the pixel compensation of Cheng et al. [12] and HE synergistically. Recently, Lee et al. [13] proposed the brightness-compensated contrast enhancement (BCCE) algorithm, which considers brightness compensation and contrast enhancement simultaneously using a Lagrangian cost function. However, we have found that HE often incurs excessive contrast increment owing to the calculations involved, irrespective of the image characteristics (e.g., dark or bright). Such a problem decreases the visual satisfaction of the resultant images. In order to overcome this problem, there have been recently conducted studies such as contrast enhancement by combining tone mapping and HE [14]. However, there remains a problem in that they are not HVS-aware.

In this paper, we propose a new dimming method that combines pixel compensation and histogram specification (HS) [10] to reflect the image characteristics fully. This method can lead to more HVS-aware results than other techniques using HE or other contrast enhancement techniques [11-14]. The proposed method conducts restricted pixel compensation to prevent image distortion, which is exposed by existing pixel compensation techniques [2-8]. If further 
restoration of the image brightness is required, additional image improvement is carried out by HS, and optimal visual satisfaction results are obtained based on the SSIM [4] index by using the parameters in the proposed HS.

The remainder of this paper is organized as follows. In Section 2, we discuss related works, while in Section 3, we explain in detail operations of the proposed algorithm. In Section 4, we present the experimental results. Finally, in Section 5, we conclude the paper.

\section{Related Work}

\subsection{Image Quality Assessment}

HVS awareness refers to the visual satisfaction gained from images in the way that human eyes see, feel, and recognize. The mean opinion score (MOS) is widely used to represent this subjective satisfaction. MOS is obtained from reviewers evaluating the target image with a score from 1 to 5 according to their visual satisfaction regarding the quality of the image. It is computed by averaging the resultant scores, considering z-score as the standard deviation. Because the measurement of MOS for the assessment of images requires considerable time and cost, objective IQA tools have been developed.

One of the most widely used IQA tools is SSIM [4], which is based on the combination of luminance, contrast, and structure, and it is designed to reflect HVS awareness. The SSIM index is calculated using mean values, variances, and the covariance of pixels from the two images $\mathrm{x}$ and $\mathrm{y}$, as shown in Eq. (1).

$$
\operatorname{SSIM}(x, y)=\frac{\left(2 u_{x} u_{y}+C_{1}\right)\left(2 \sigma_{x y}+C_{2}\right)}{\left(u_{x}{ }^{2}+u_{x}{ }^{2}+C_{1}\right)\left(\sigma_{x}{ }^{2}+\sigma_{x}{ }^{2}+C_{2}\right)}
$$

In Eq. (1), the SSIM index ranges from 0 to 1, and the closer the index is to 1, the more similar $\mathrm{x}$ and $\mathrm{y}$ are. Fig. 1 shows the SSIM indices for images (b) and (c), which are dimmed 0.9 and 0.7 times respectively, compared with the original image (a). If the degree of dimming is small, as in (b), the SSIM index is close to 1. In the case of (c) with more dimming, a lower value of 0.9097 is obtained and the resultant image is clearly distorted against (a). SSIM has the merit of enabling IQA numerically, and it is widely used as it considers the HVS.

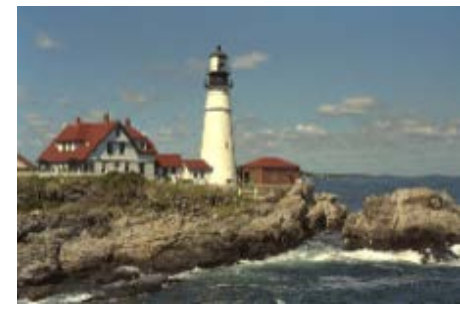

(a) Original

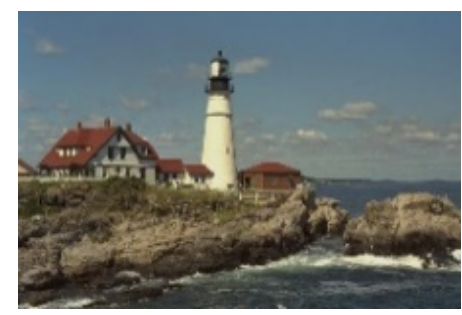

(b) Dimmed $(\times 0.9)$ $\operatorname{SSIM}(a, b)=0.9909$

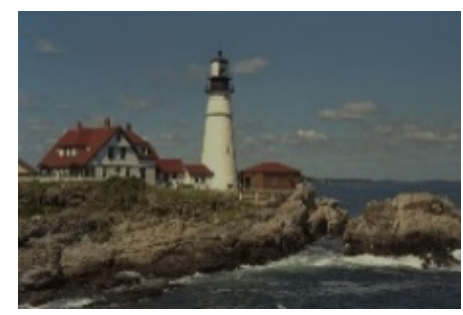

(c) Dimmed $(\times 0.7)$ $\operatorname{SSIM}(\mathrm{a}, \mathrm{c})=0.9097$

Fig. 1. SSIM indices for two dimmed images. 


\subsection{Pixel Compensation}

In TFT-LCDs, power consumption is much more greatly affected by the backlight intensity than the other components [1]. Thus, many techniques employ backlight dimming for power saving, while restoring visual luminance through pixel compensation. Pixel compensation scales the pixel value up to the desired brightness level, and its equation is given by the maximum brightness level of backlight $B_{\max }$ and the dimmed brightness $B_{\text {diimmed }}$ in Eq. (2):

$$
y(i, j)=x(i, j) \times \frac{B_{\max }}{B_{\text {diimmed }}}
$$

where $\mathrm{i}$ and $\mathrm{j}$ are the location indices of a pixel and $B_{\max } / B_{\text {diimmed }}$ refers to the compensation factor, which is proportional to the dimming ratio. Because the maximum pixel value is limited to a specific value (e.g., 255 for 8 bits), if large-scale pixel compensation is carried out, clipping artifacts occur as a result of a large number of saturated pixels at the maximum pixel value, as shown in Fig. 2. The more the number of clipping artifacts, the greater will be the color distortion that appears on the display. To minimize color distortion due to these artifacts, Cheng et al. [12] proposed a dimming method that stretches the middle-level band in images. However, this method is rarely used, as it does not perform well in terms of brightness compensation.

Pixel compensation is widely used in dimming algorithms owing to its simple computation and significant improvement of visual satisfaction. However, pixel saturation often incurs blurred structures in addition to color distortion, as shown in Fig. 2. To solve this problem, recent research has specified an allowable error based on the IQA indices. For example, Kang et al.'s method [2] executes SSIM-based backlight dimming using pixel compensation to obtain a resultant image that is close to the target quality given by a user. However, this method largely focuses on the mitigation of distortion, as opposed to power saving.

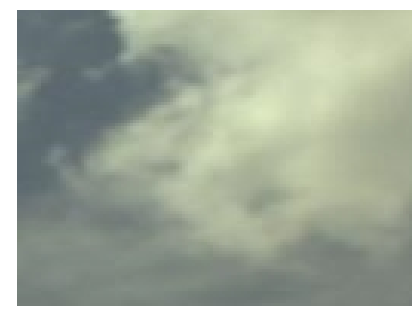

(a) Original

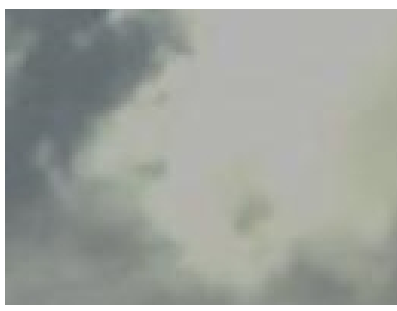

(b) After pixel compensation

Fig. 2. Example of clipping artifacts from pixel compensation.

\subsection{Histogram Equalization}

HE [10] enhances the contrast by using a cumulative distribution function (CDF) that is based on the histogram of an image. Because backlight dimming decreases the image contrast, HE is frequently used to compensate for this. HEBS [10] is a well-designed, low-power method based on HE. HEBS combines HE and a contrast enhancement pixel compensation technique [12], improving the image quality by increasing the contrast and brightness concurrently. However, HE usually conducts histogram stretching, and does not consider the color and 
brightness information of an image. Thus, it often produces a distortion of average brightness and color, and fails to enhance contrast naturally.

To preserve image brightness, brightness-preserving bi-histogram equalization (BBHE) [15] and dualistic sub-image histogram equalization (DSIHE) [16] divide a histogram into two sub-histograms and equalize each of them. Recently, techniques for controlling the degree of the contrast ratio have been developed, such as the histogram modification framework (HMF) [17]. However, many methods still execute processing, independent of the image characteristics, and they incur a large amount of computational overhead, making existing HE methods inappropriate for the image compensation included in backlight dimming.

\subsection{Histogram Specification}

HS [10] transforms the original CDF of an image into the desired CDF, which can be obtained by integrating the required histogram after some manipulation of the original $\mathrm{CDF}$. Let the CDF of an input image be $c_{i}$, and the desired CDF be $c_{d}$. Then, the HS is calculated as follows:

$$
\operatorname{result}\left(k_{a}\right)=\left\{k_{b} \mid \min \left(c_{i}\left(k_{a}\right)-c_{d}\left(k_{b}\right)\right)\right\}
$$

where $\operatorname{result}\left(k_{a}\right)$ is a transformation function for mapping to the resultant pixel value. While HE modifies the original histogram, independent of the image characteristics, HS can reflect user requirements through modifying the image histogram, and this can be done by calculating the desired histogram or the desired CDF.

\section{Proposed Method}

In this study, we propose a novel dimming technique to obtain both high power saving and HVS-awareness by combining pixel compensation and HS. The overall flow chart of the proposed method is shown in Fig. 3, and the major processes are described in each of the relative subsections. Using the proposed method, we first executed backlight dimming, and a color image is converted into a grayscaled image, as explained in subsection 3.1. Subsequently, we performed restricted pixel compensation to minimize the image distortion, as explained in subsection 3.2. We obtain the clipping point from a CDF and a power saving ratio to prevent clipping artifacts, as shown in Fig. 2, by setting the threshold of the ratio of saturated pixels to $r_{\max }$ after pixel compensation. If the current backlight intensity is sufficiently large, and thus the ratio of the saturated pixels after pixel compensation does not exceed $r_{\max }$, the clipping point is proportional to the backlight intensity. In this case, we assume that the input image is restored to sufficient brightness by using restricted pixel compensation. Otherwise, the clipping point is only affected by $r_{\max }$ and the result of restricted pixel compensation is not proportional to the backlight intensity. In this case, the HS-based search algorithm or the HS-based parameter-computing algorithm (HSPCA) described in subsections 3.4 and 3.5 are executed because the result of the restricted pixel compensation does not recover to a sufficient image brightness. The proposed algorithms calculate the desired CDF using the $\mathrm{CDF}$ of the input image so that they can perform image compensation that reflects the image characteristics. Finally, the resultant grayscaled image is converted into a color image to the 
same degree as the difference between two grayscaled images. This is explained in subsection 3.6.

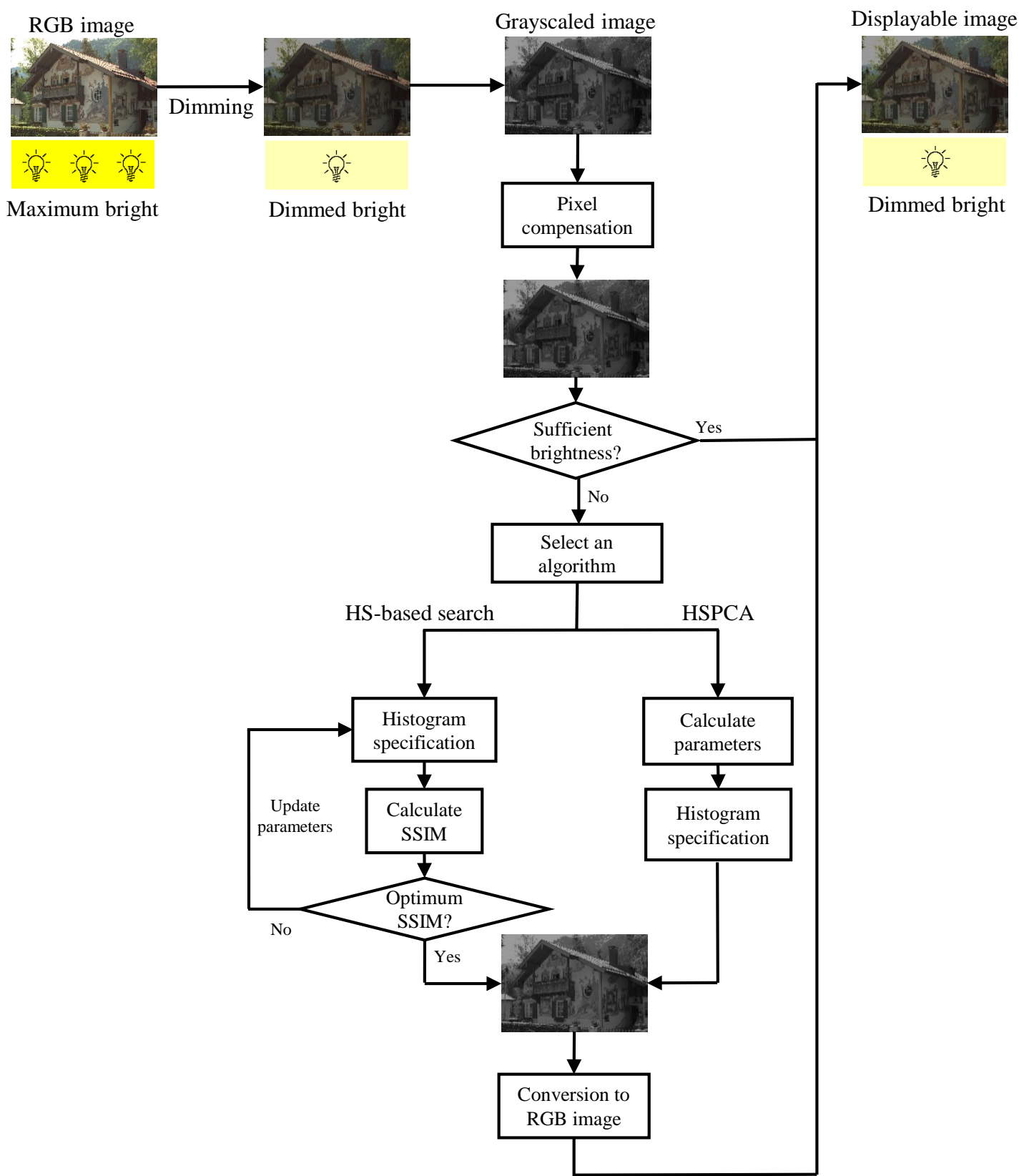

Fig. 3. Overall flowchart of the proposed method. 


\subsection{Conversion to a grayscaled image}

Various kinds of information are distorted owing to backlight dimming such as contrast ratio and color. Among them, the loss of luminance is greatest. In order to consider the image brightness, we convert a color image into a grayscaled image using Eq. (4).

$$
x(i, j)=0.2989 * I_{R}(i, j)+0.5870 * I_{G}(i, j)+0.1140 * I_{B}(i, j)
$$

Here, $I_{R}, I_{G}$, and $I_{B}$ refer to the R, G, and B channels of the color image, respectively, and $x$ refers to the pixel value of the grayscaled image. This enables us to focus on the luminance information of the image and reduce the computational cost of the proposed method because it reduces the number of calculation dimensions.

\subsection{Restricted Pixel Compensation}

This procedure restricts the restoration of image brightness by limiting the maximum number of saturated pixels in order to minimize the clipping artifacts shown in Fig. 2. After an input color image is converted into a grayscaled image, a CDF is first generated, as in Eq. (5), using the histogram h and total pixel number $N$ of the grayscaled image.

$$
c_{i}(k)=\frac{1}{N} \sum_{a=0}^{k} h(a)
$$

Next, the threshold of the ratio of saturated pixels and maximum pixel value are set to $r_{\max }$ and $k_{\max }$, respectively. We assume $k_{\max }=255$ in this paper. Then, a clipping point $P$ is given as follows:

$$
P=\max \left\{c_{i}^{-1}\left(1-r_{\max }\right), 255 \times\left(\frac{B_{\text {diimmed }}}{B_{\max }}\right)\right\} \text {. }
$$

Here, $P$ is determined by the dimmed brightness $B_{\text {diimmed }}$ and $r_{\max }$. In Eq. (6), $C_{i}^{-1}\left(1-r_{\max }\right)$ is the pixel value when the CDF of an image is $1-r_{\max }$, and it is not affected by $B_{\text {diimmed }}$. Then, $255 \times\left(B_{\text {diimmed }} / B_{\max }\right)$ is proportional to $B_{\text {diimmed }}$. When $B_{\text {diimmed }}$ is sufficiently large, $P$ becomes $255 \times\left(B_{\text {diimmed }} / B_{\max }\right)$, and in the opposite case, $P$ becomes $c_{i}^{-1}\left(1-r_{\max }\right)$. Then, if $r_{\max }$ gets smaller, $c_{i}^{-1}\left(1-r_{\max }\right)$ becomes larger and thus $B_{\text {diimmed }}$ becomes larger in case $c_{i}^{-1}\left(1-r_{\max }\right)=255 \times\left(B_{\text {diimmed }} / B_{\max }\right)$. Because the clipping point is the minimum value of the pixels that will be saturated, the pixel domain below the clipping point is stretched as follows:

$$
y_{p}(i, j)=\left\{\begin{array}{lll}
x(i, j) \times \frac{255}{P} & \text { subject to } x(i, j)<P \\
255 & \text { subject to } P \leq x(i, j)
\end{array}\right.
$$


When the current backlight intensity $B_{\text {dimmed }} / B_{\max }$ is larger than $c_{i}{ }^{-1}\left(1-r_{\max }\right) / 255$, a large amount of distortion is not expected while computing Eqs. (6) and (7), and pixel compensation is applied according to the amount of dimming. Otherwise, restricted pixel compensation is applied according to the threshold of the ratio of saturated pixels $r_{\max }$. Fig. 4 shows the result of pixel compensation with $B_{\text {diimmed }} / B_{\max }=0.7, r_{\max }=0.05$, and it can be seen that clipping artifacts are mitigated owing to protection against excessive saturation.

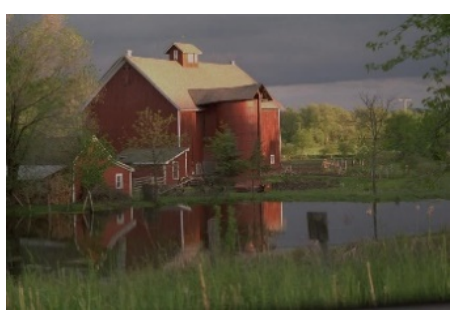

(a) Original

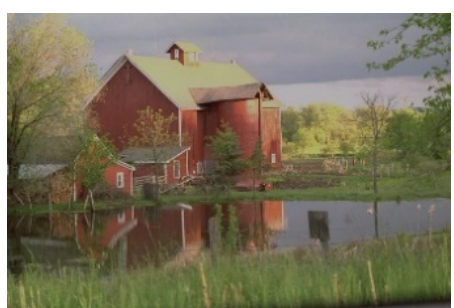

(b) After execution

Fig. 4. Result of restricted pixel compensation.

If the clipping point becomes the minimum value, and the brightness of the restored image is therefore not sufficient, an HS-based search or the HSPCA, as explained in Subsections 3.3 and 3.4, is executed. If the restored image has a sufficient brightness, the resultant image will be displayed without conducting HS.

\subsection{Histogram Specification}

In this study, HS is executed using the algorithms described in Subsections 3.3 and 3.4, and it enables image compensation that is dependent on the image characteristics by obtaining the desired CDF, using the CDF of an image that has not had its brightness restored through pixel compensation. In order to minimize color distortion, the desired CDF is divided into three regions, using two thresholds.

First, to calculate the desired $\mathrm{CDF}$, the $\mathrm{CDF} c_{p}$ of the image resulting from the pixel compensation is calculated using Eq. (8).

$$
c_{p}(k)=\frac{1}{N} \sum_{a=0}^{k} h_{p}(a)
$$

Here, $h_{p}$ is the histogram of $y_{p}$ in Eq. (7). Pixel compensation that is dependent on the image characteristics is derived by calculating the desired CDF using $c_{p}$ in Eq. (8). Given the thresholds $T_{1}$ and $T_{2}\left(T_{1}<T_{2}\right)$, the desired CDF is divided into three equal regions based on these thresholds. This has the effect of suppressing excessive brightness change and color distortion. Fig. 5 shows the effects of HS when no threshold is used (b), and when a single threshold (c) and two thresholds (d) are used. In this figure, it can be seen that if the desired CDF is divided using an appropriate number of thresholds, we can obtain the natural image brightness while incurring less color distortion. 


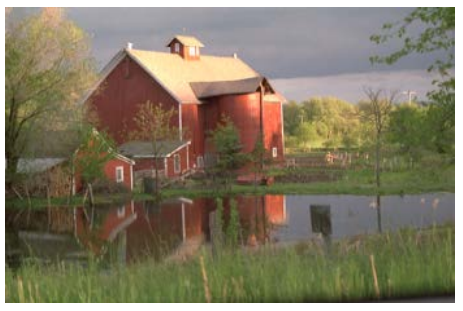

(a) Original

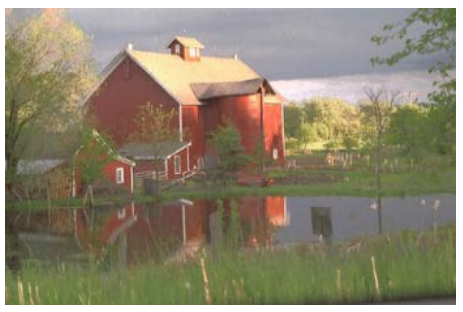

(c) HS result using one threshold

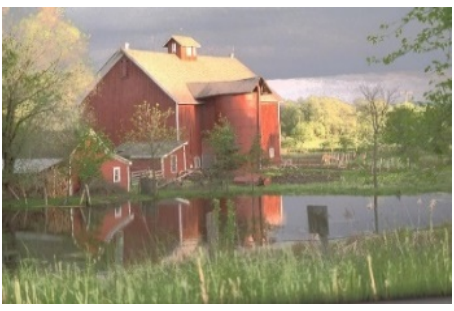

(b) HS result without using a threshold

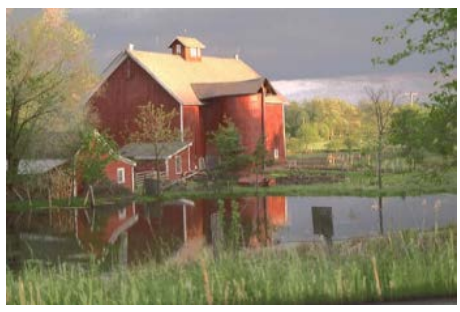

(d) HS result using two thresholds

Fig. 5. Effects of HS without/with region division.

Next, the HS is processed per region. For the region where the pixel value is less than $T_{1}$, we calculate the desired CDF $c_{d}$ using Eq. (9).

$$
c_{d}(k)=c_{p}\left(T_{1}\right) \times\left(\frac{c_{p}(k)}{c_{p}\left(T_{1}\right)}\right)^{a} \quad \text { subject to } k<T_{1}
$$

Here, $\alpha$ is a parameter that can control the pixel brightness in this region, and it is larger than 0. If this value becomes large, the image brightness increases after HS is applied, as shown in Fig. 6. Because $c_{p}\left(T_{1}\right)$ indicates the maximum value in the $k<T_{1}$ region, when $k$ approaches $T_{1}$ in Eq. (9), the result of the pixel compensation will be maintained. Therefore, color distortion can be minimized when HS is executed according to Eq. (3).

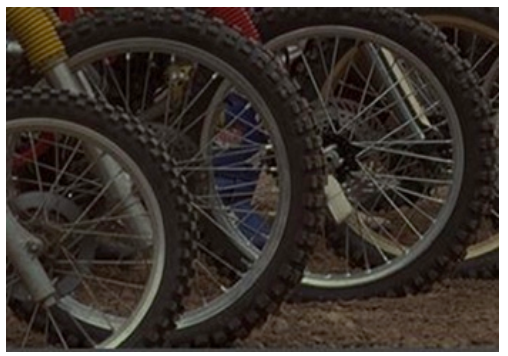

(a) Original

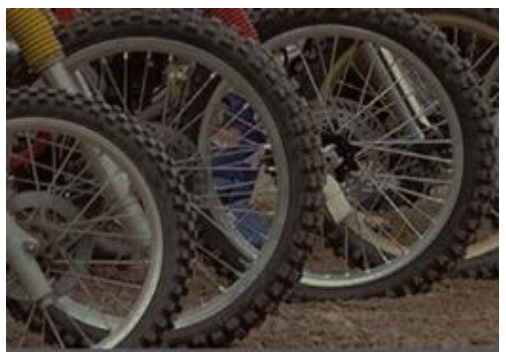

(b) After execution

Fig. 6. Effect of HS using the parameter $\alpha$. 
For the region where the pixel value is between $T_{1}$ and $T_{2}$, we calculate the desired CDF $c_{d}$ using Eq. (9).

$$
\begin{aligned}
& A=\frac{c_{p}(k)-c_{p}\left(T_{1}\right)}{c_{p}\left(T_{2}\right)-c_{p}\left(T_{1}\right)} \\
& B=c_{p}(k)-c_{p}\left(T_{1}\right) \\
& c_{d}(k)=c_{p}\left(T_{1}\right)+B \times A^{\beta} \quad \text { subject to } T_{1} \leq k \leq T_{2}
\end{aligned}
$$

Here, $A$ is the ratio of the proportion of pixels that exist between $T_{1}$ and $k$ to the proportion of pixels that exist between $T_{1}$ and $T_{2}$, and $B$ is the proportion of pixels that exist between $T_{1}$ and $T_{2}$ over the total number of pixels. $\beta$ is another parameter that can control the pixel brightness, and it is larger than 0 like $\alpha$. When the pixel value $k$ lies between $T_{1}$ and $T_{2}, c_{d}$ is a monotonically increasing function; thus, $c_{d}$ is designed to have a value between $c_{p}\left(T_{1}\right)$ and $c_{p}\left(T_{2}\right)$ in the last equation in Eq. (10).

For the remaining region, where the pixel value is larger than $T_{2}$, we calculate $c_{d}$ using Eq. (11).

$$
\begin{aligned}
& C=\frac{c_{p}(k)-c_{p}\left(T_{2}\right)}{1-c_{p}\left(T_{2}\right)} \\
& D=1-c_{p}\left(T_{2}\right) \\
& c_{d}(k)=c_{p}\left(T_{2}\right)+D \times C^{\chi} \quad \text { subject to } T_{2} \leq k
\end{aligned}
$$

where $C$ is the ratio of the proportion of pixels that exist between $T_{2}$ and $k$ to the proportion of pixels larger than $T_{2}$, and $\mathrm{D}$ is the ratio of the proportion of pixels larger than $T_{2}$ to the total number of pixels. $\gamma$ controls the pixel brightness similarly to $\alpha$ and $\beta$, and it is larger than $0 . c_{d}$ is calculated using the last equation in Eq. (11), and it is a monotonically increasing function.

Using $c_{d}$ calculated from Eqs. (9), (10), and (11), we perform the following comparison.

$$
k_{\text {result }}\left(k_{a}\right)=\left\{k_{b} \mid \min \left(c_{p}\left(k_{a}\right)-c_{d}\left(k_{b}\right)\right)\right\}
$$

In Eq. (12), $k_{\text {result }}$ is a transformation function that conducts mapping from $k_{a}$, which is the ratio of the pixel value of the image $y_{p}$ resulting from pixel compensation to the resultant pixel value $k_{b}$. This transformation function maps the input pixel value $y_{p}(i, j)$ to the output pixel value $y_{\text {result }}(i, j)=k_{\text {result }}\left(y_{p}(i, j)\right)$. Thus, the resultant image is given by

$$
y_{\text {result }}(i, j)=k_{\text {result }}\left(y_{p}(i, j)\right) .
$$




\subsection{HS-based Search Algorithm}

If $\mathrm{P}$ is not the minimum value $c_{i}^{-1}\left(1-r_{\max }\right)$ in Eq. (6), the pixel compensation in Eq. (7) can restore most of the brightness with minimal image distortion, and thus no additional compensation is required. However, if $P$ reaches the minimum value, restricted pixel compensation occurs; thus, additional processing is required to enhance the image quality. In this paper, we employ the parameters $\alpha, \beta$, and $\gamma$ to achieve additional visual satisfaction. To this end, we propose a search algorithm using SSIM to find the optimal parameters, as shown in Fig. 7. First, the algorithm initializes $\alpha, \beta$, and $\gamma$ to zeros and executes HS. Then, the SSIM index for the original and resultant images is evaluated. If the SSIM index can be increased, the optimal HVS-aware resultant image is searched, along with some variations in the parameters.

\subsection{HS-based Parameter Computation Algorithm}

While the search algorithm in Fig. 7 can obtain the optimal HVS-aware image based on the SSIM index, it requires considerable execution time. Thus, we propose the faster HSPCA, which estimates parameters $\alpha, \beta$, and $\gamma$ considering the operational features for the desired CDF and histogram in Fig. 8.

When HS is executed in the HS-based search algorithm, the pixel value changes in proportion to the difference between $c_{p}\left(k_{a}\right)$ and $c_{d}\left(k_{b}\right)$, as shown in Eq. (12). Furthermore, because the desired CDF is an exponential function, the variation in pixel values in Eqs. (12) and (13) is large in the middle of each of the three regions. Next, we designed a new algorithm to reduce the computational overhead by calculating the required parameters $\alpha_{r}, \beta_{r}$, and $\gamma_{r}$ in Eqs. (14), (15), and (16), respectively.

$$
\begin{aligned}
& \alpha_{r}=C_{\alpha} \times\left(\frac{P}{255}-\frac{B_{\text {diimmed }}}{B_{\max }}\right) \times \frac{\sum_{a=21}^{T_{1}-20} h_{p}(a)}{\sum_{a=0}^{T_{1}} h_{p}(a)} \\
& \beta_{r}=C_{\beta} \times\left(\frac{P}{255}-\frac{B_{\text {diimmed }}}{B_{\max }}\right) \times \frac{\sum_{a=T_{1}+21}^{T_{2}-20} h_{p}(a)}{\sum_{a=T_{1}}^{T_{2}} h_{p}(a)} \\
& \gamma_{r}=C_{\gamma} \times\left(\frac{P}{255}-\frac{B_{\text {diimmed }}}{B_{\max }}\right) \times \frac{\sum_{a=T_{2}+21}^{255} h_{p}(a)}{\sum_{a=T_{2}}^{255} h_{p}(a)}
\end{aligned}
$$

Here, $C_{\alpha}, C_{\beta}$, and $C_{\gamma}$ are constants for the optimal parameters and $(P / 255)-\left(B_{\text {diimmed }} / B_{\max }\right)$ indicates the degree of restoration against the required brightness after pixel compensation. Each parameter is proportional to the ratio of the pixels in the middle of each region. For example, in Eq. (14), $\alpha_{r}$ is the parameter of the $k<T_{1}$ region and $\sum_{a=21}^{T_{1}-20} h_{p}(a)$ is the number of pixels in the middle of this region. Thus, $\alpha_{r}$ is proportional to $\sum_{a=21}^{T_{1}-20} h_{p}(a) / \sum_{a=0}^{T_{1}} h_{p}(a)$. 


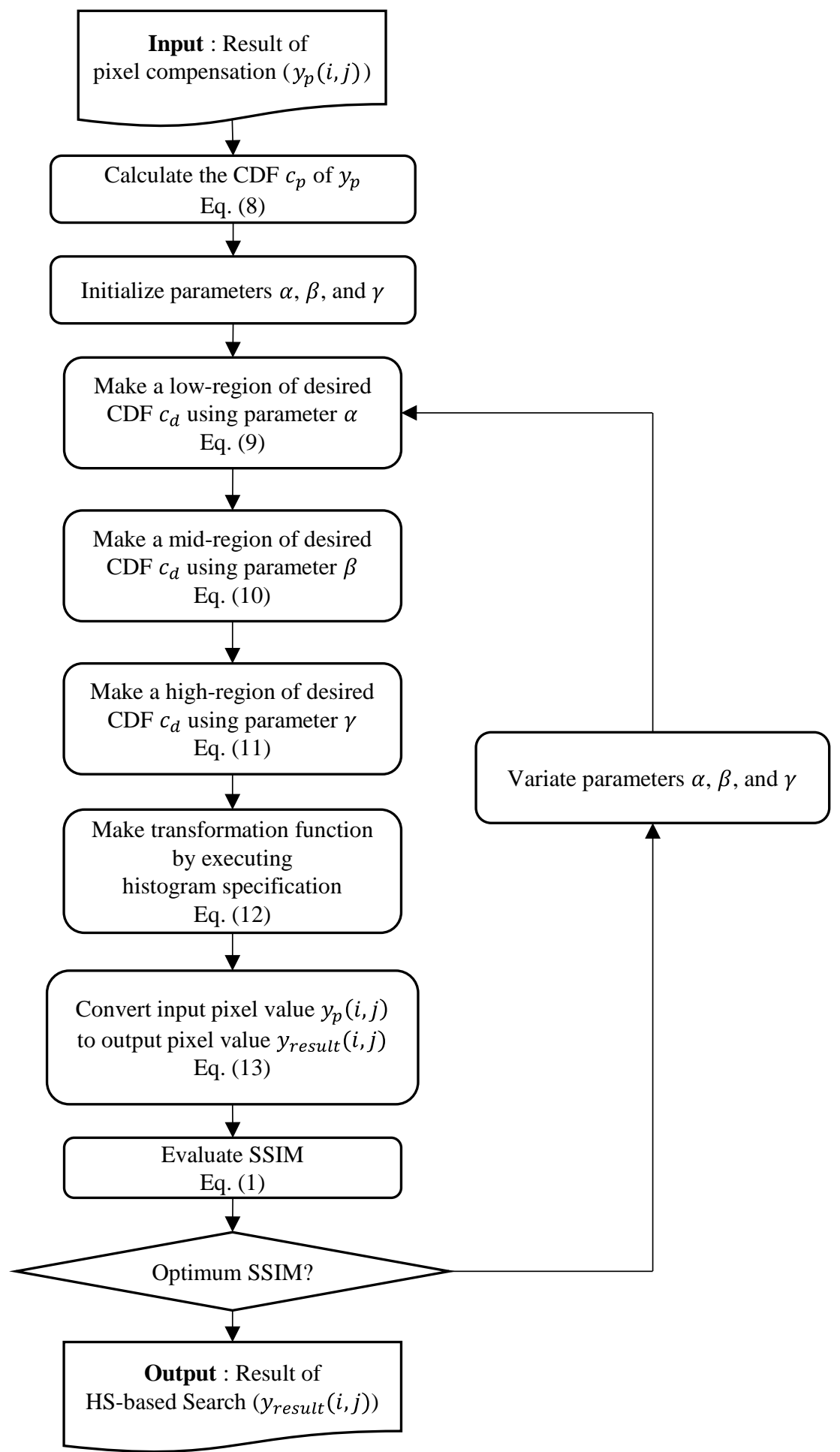

Fig. 7. Flowchart of the HS-based search algorithm. 


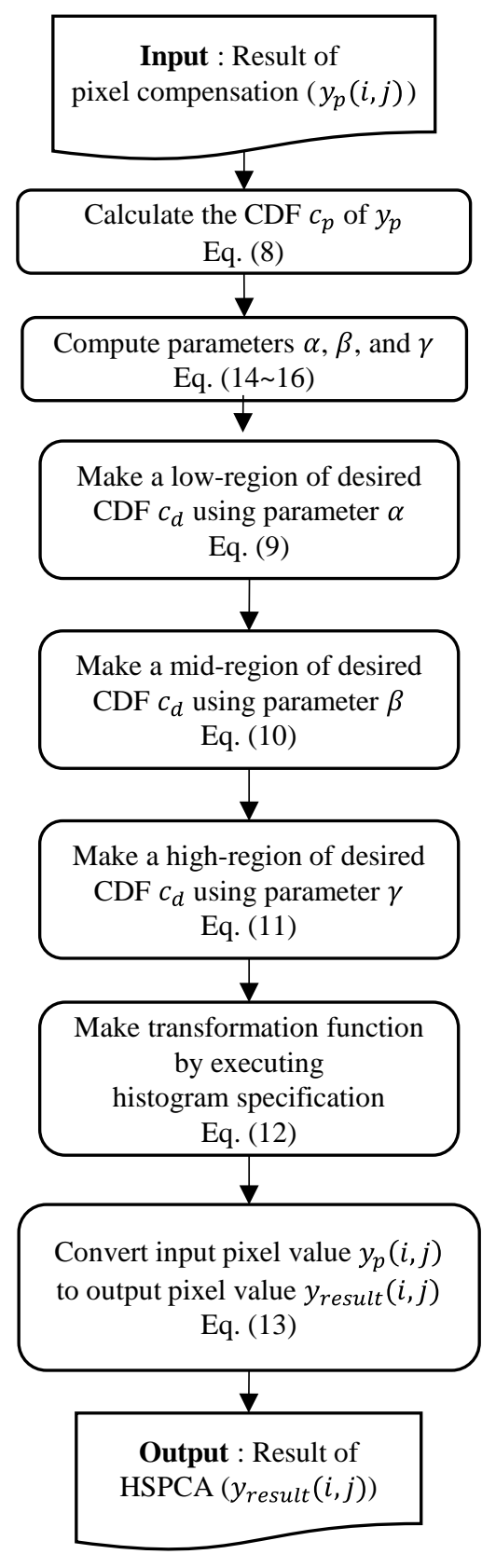

Fig. 8. Flowchart of the HSPCA.

By calculating parameters $\alpha, \beta$, and $\gamma$, as in Eqs. (14)-(16), the HSPCA does not need the search algorithm shown in Fig. 7. The algorithm in Fig. 7 has a high computational cost because HS computes the desired CDF and performs various operations until it updates the pixel value and the SSIM repeats window operations for all pixels. In addition, such processes are repeated many times and they are not practical. Unlike this algorithm, the HSPCA can find the optimal desired CDF by performing only a single HS process, and it is therefore possible to save a great deal of computational cost compared to the search algorithm in Fig. 7. 


\subsection{Conversion to an RGB image}

Because all of the previously described techniques have been applied to a grayscaled image, we convert this image to an RGB color image. To this end, we multiply each color channel of the input image proportionally by the variation of the grayscaled pixel level in Eq. (17).

$$
\begin{aligned}
& S_{R}(i, j)=I_{R}(i, j) \times \frac{y_{\text {result }}(i, j)}{x(i . j)} \\
& S_{G}(i, j)=I_{G}(i, j) \times \frac{y_{\text {result }}(i, j)}{x(i . j)} \\
& S_{B}(i, j)=I_{B}(i, j) \times \frac{y_{\text {result }}(i, j)}{x(i . j)}
\end{aligned}
$$

where $S_{R}, S_{G}$, and $S_{B}$ are the R, G, and B color channels of a displayed image, respectively, $I_{R}, I_{G}$, and $I_{B}$ are the $\mathrm{R}, \mathrm{G}$, and $\mathrm{B}$ color channels of an input image, respectively, and $x, y_{\text {result }}$ is the pixel value of a grayscaled image, where $y_{\text {result }}$ is the result of Eq. (13).

\section{Experimental Results}

For the evaluation, we conducted the experiments using the LIVE database [18] and MATLAB 2014b on a desktop computer with an i7-3770 3.40 GHz CPU and 16.0 GB DRAM. Throughout the experiments, we used parameter settings of $r_{\max }=0.05, T_{1}=85$, and $T_{2}=170$. The LIVE database consists of 29 images and various kinds of natural images, as shown in Fig. 9.

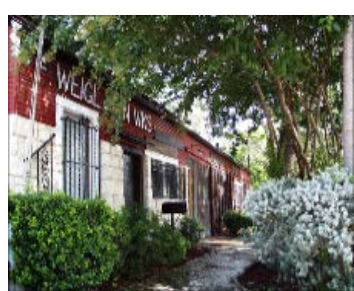

(a) building2

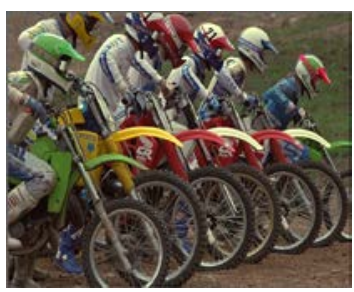

(e) bikes

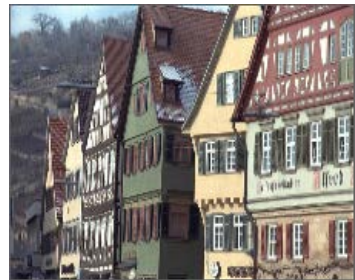

(b) buildings

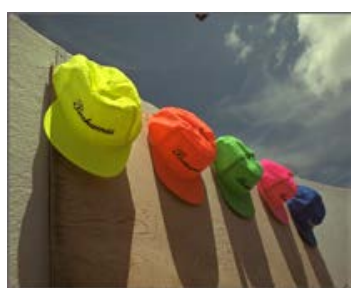

(f) caps

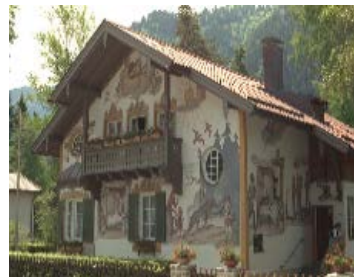

(c) paintedhouse

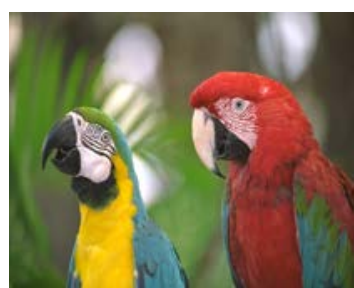

(g) parrots

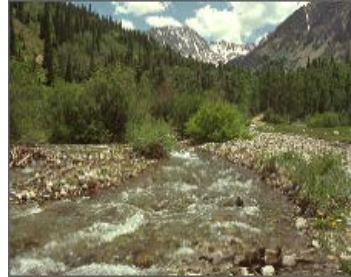

(d) stream

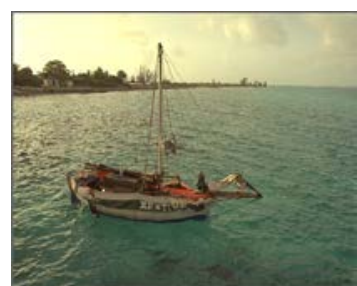

(h) sailing1

Fig. 9. Some of the LIVE database images [18]. 
We conducted the experiments using the proposed algorithms while the backlight was dimmed by 0.7 to 0.9 times relative to the maximum brightness. Fig. 10 and Fig. 11 show the results of HEBS, BCCE, the HS-based search algorithm illustrated in Fig. 7, and the HSPCA shown in Fig. 8, using images in Fig. 9, where the brightness is fixed to 0.7 times that of the original image.

In Fig. 10, the results of HEBS, BCCE, the HS-based search algorithm, and the HSPCA appear distinctly brighter than the dimmed images (e)-(h), respectively. However, in the HEBS results (i) and (j), certain parts of the structures are blurred, including the flowers. Furthermore, (k) and (l) show a large degree of color distortion because of overstretching contrast, and (m-p) these are the results of BCCE, which are more blurred than the HEBS results. On the contrary, the qualities of (q)-(x), which are the results of the proposed methods, are naturally enhanced in comparison with (e)-(h). These results can be verified by using IQA tests. The SSIM indices of HEBS and BCCE are lower than those of the HS-based search algorithm. In comparison with the IQA values given in Fig. 1, it can be seen that the above-mentioned differences in the SSIM indices are significant.

In Fig. 11, we compare existing and proposed algorithms for four other different images of the LIVE database. As with the results in Fig. 10, HEBS and BCCE appear to be clearly brighter than the dimmed images (e)-(h). However, the results of HEBS show poor visual satisfaction because of the excessive increase of contrast, as shown in (i)-(l). In the results of HEBS, (i)-(j) are overly bright owing to histogram overstretch, and (k)-(l) show remarkably dark areas. In addition, the results of BCCE show a smaller increase in contrast compared to HEBS, but there remains a large increase in contrast and blurring. (m)-(p) are not much brighter than (i)-(l), but we observe blurs at the rider's jacket, the yellow cap, and the abdomen of the bird in (m), (n), and (k), respectively. By contrast, the proposed methods achieved a natural quality enhancement, as observed in the results in Fig. 10. The SSIM indices of HEBS and BCCE are lower than those of the HS-based search algorithm. In particular, the HS-based search algorithm has a visual satisfaction that is up to 0.14 of the SSIM index compared with existing techniques. These results indicate that excessive contrast enhancement has a negative influence on actual visual satisfaction.

In Fig. 10 and Fig. 11, we observe that the difference in SSIM indices between the HS-based search algorithm and the HSPCA is negligible. In terms of computation time, the HS-based search algorithm takes approximately 10 seconds, while the HSPCA takes approximately 0.35 seconds per image. Based on these results, we believe that the HSPCA achieves a computation speed of approximately 28.6 times that of the HS-based search algorithm, while maintaining a very similar image quality.

Table 1 shows the average of the SSIM indices, which we obtained by using all of the LIVE database images for the various dimming methods according to different backlight dimming ratios. For the same dimming ratios, HEBS and BCCE show lower visual satisfaction, while the proposed methods achieve high SSIM indices. Moreover, the SSIM indices of HEBS and BCCE are much lower than that of the dimmed result. The SSIM index of the HS-based search algorithm is up to $15.9 \%$ larger than that of HEBS, $12.8 \%$ than that of BCCE, and $6.2 \%$ than that of the dimmed result. This means that the HS-based search algorithm achieves better visual satisfaction than the dimmed result and a much superior visual satisfaction to HEBS and BCCE. The maximum difference in SSIM values between the HS-based search algorithm and the HSPCA is 0.004 , which means that the HSPCA can estimate the optimum parameters of the HS-based search algorithm effectively. 

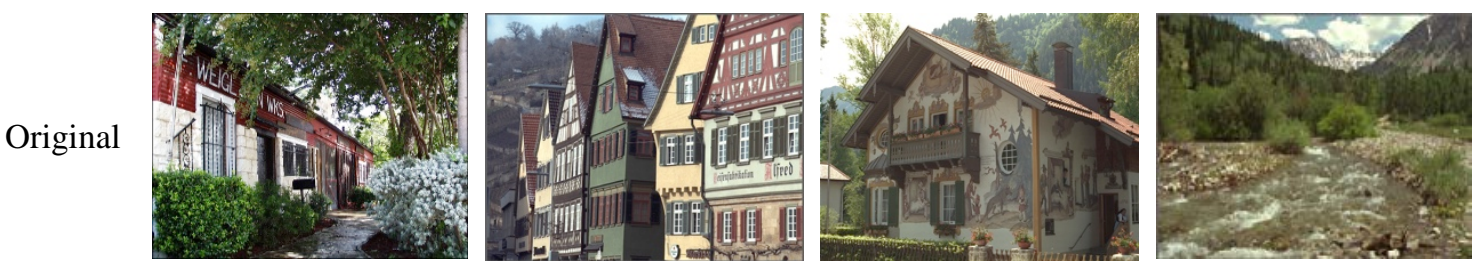

(a) $\operatorname{SSIM}(a, a)=1.0000$ (b) $\operatorname{SSIM}(b, b)=1.0000$
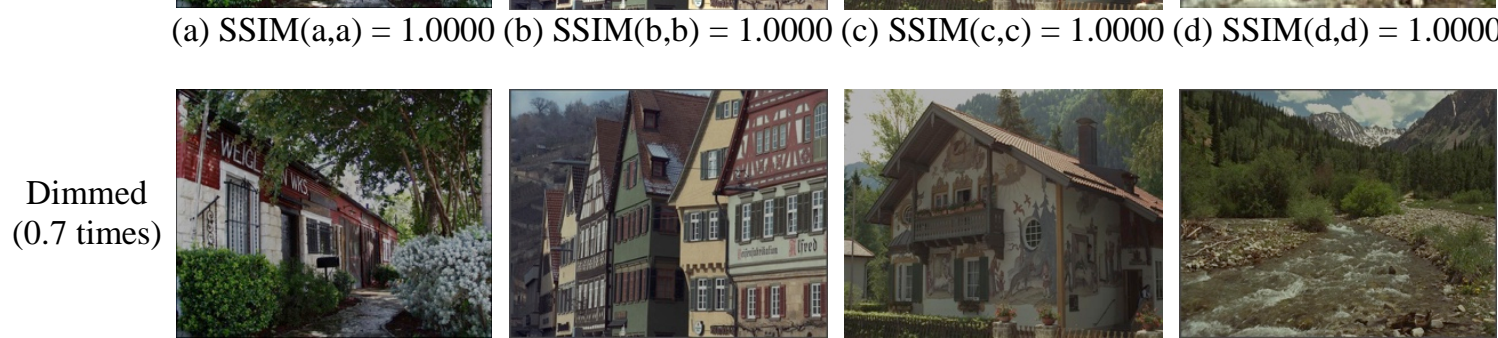

(e) $\operatorname{SSIM}(a, e)=0.8879$ (f) $\operatorname{SSIM}(b, f)=0.8926$
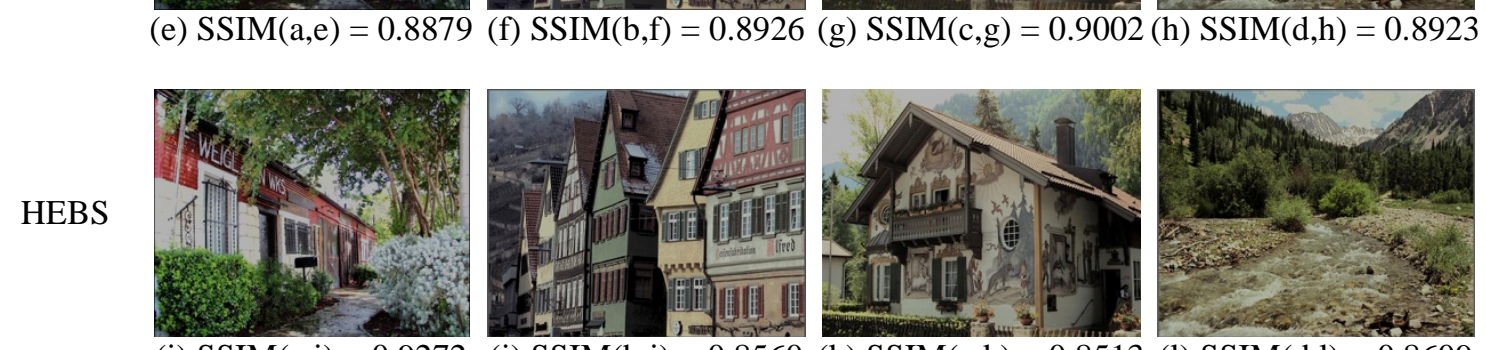

(i) $\operatorname{SSIM}(\mathrm{a}, \mathrm{i})=0.9272$

(j) $\operatorname{SSIM}(b, j)=0.8560$

$(\mathrm{k}) \operatorname{SSIM}(\mathrm{c}, \mathrm{k})=0.8513$

(l) $\operatorname{SSIM}(\mathrm{d}, \mathrm{l})=0.8609$
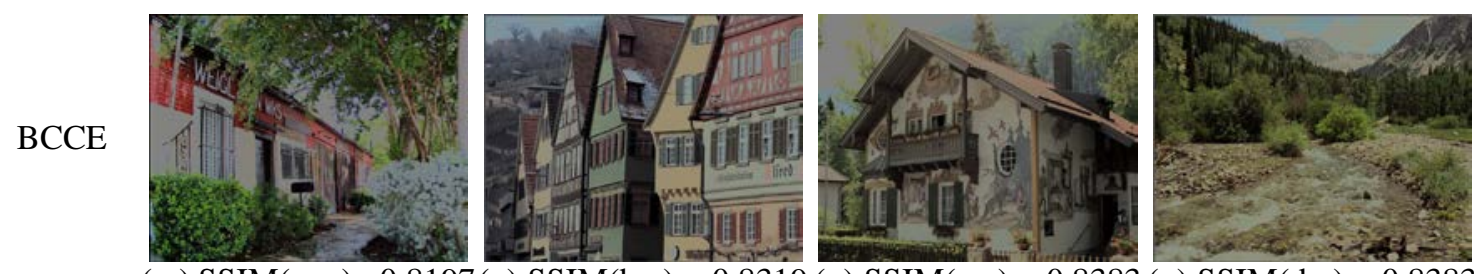

(m) $\operatorname{SSIM}(\mathrm{a}, \mathrm{m})=0.8197(\mathrm{n}) \operatorname{SSIM}(\mathrm{b}, \mathrm{n})=0.8319$

$9(\mathrm{o}) \operatorname{SSIM}(\mathrm{c}, \mathrm{o})=0.8383(\mathrm{p}) \operatorname{SSIM}(\mathrm{d}, \mathrm{p})=0.8383$
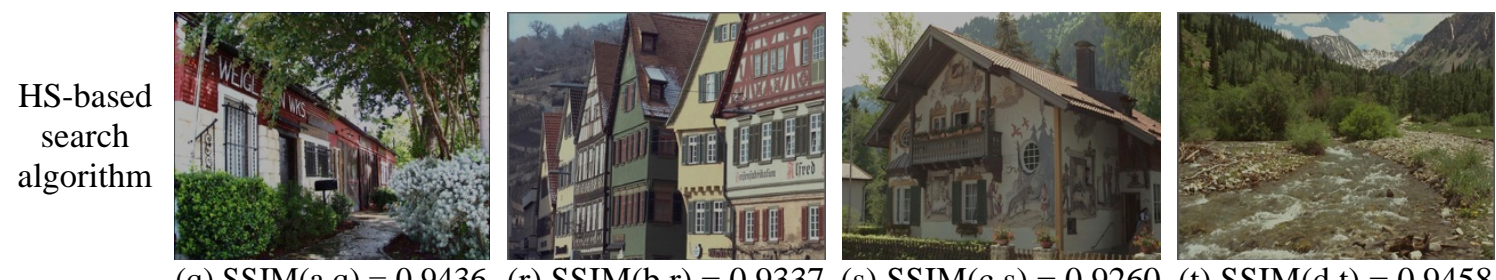

(q) $\operatorname{SSIM}(\mathrm{a}, \mathrm{q})=0.9436$
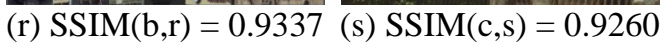

$(t) \operatorname{SSIM}(d, t)=0.9458$
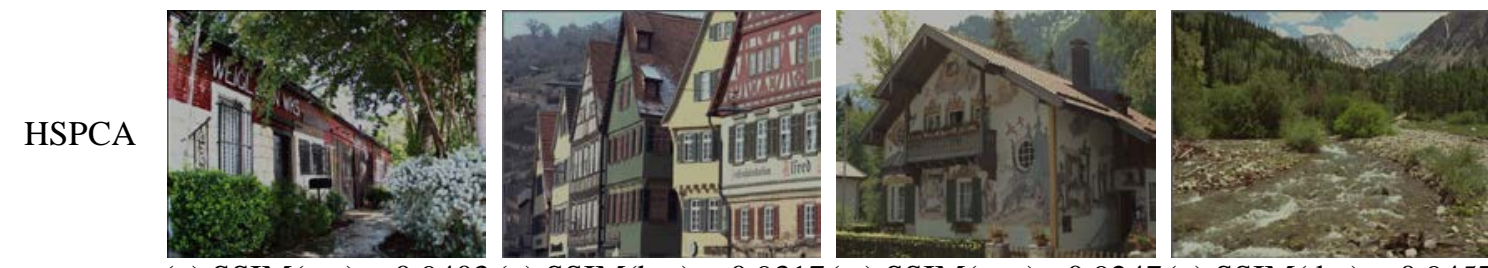

(u) $\operatorname{SSIM}(\mathrm{a}, \mathrm{u})=0.9403(\mathrm{v}) \operatorname{SSIM}(\mathrm{b}, \mathrm{v})=0.9317(\mathrm{w}) \operatorname{SSIM}(\mathrm{c}, \mathrm{w})=0.9247(\mathrm{x}) \operatorname{SSIM}(\mathrm{d}, \mathrm{x})=0.9457$

Fig. 10. Experimental results for (a)-(d) in Fig. 9 with the backlight dimmed to 0.7 times the maximum brightness. 
Original
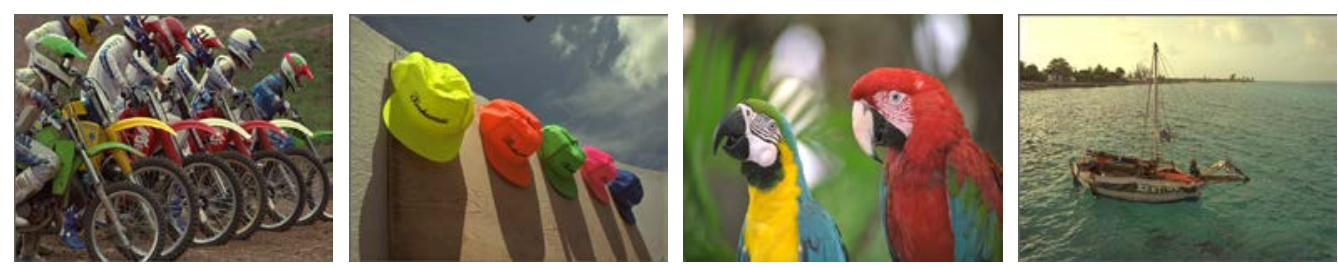

(a) $\operatorname{SSIM}(a, a)=1.0000$ (b) $\operatorname{SSIM}(b, b)=1.0000$

(c) $\operatorname{SSIM}(c, c)=1.0000(\mathrm{~d}) \operatorname{SSIM}(\mathrm{d}, \mathrm{d})=1.0000$

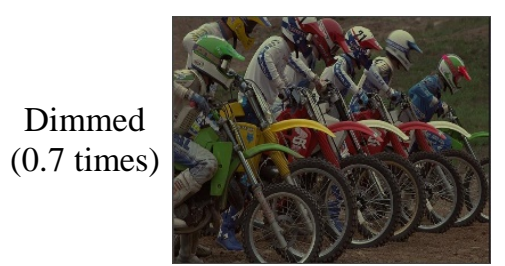

(e) $\operatorname{SSIM}(\mathrm{a}, \mathrm{e})=0.8918$

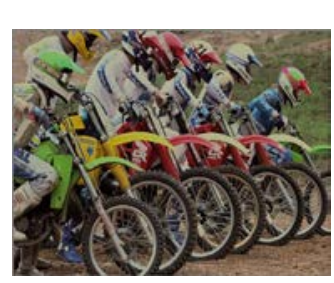

(i) $\operatorname{SSIM}(\mathrm{a}, \mathrm{i})=0.9139$

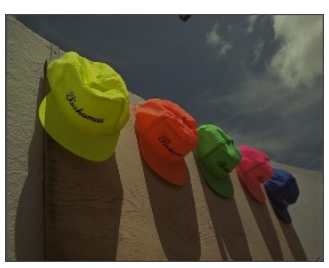

(f) $\operatorname{SSIM}(b, f)=0.9176$

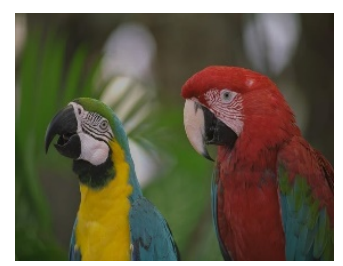

(g) $\operatorname{SSIM}(c, g)=0.9170(h) \operatorname{SSIM}(d, h)=0.9051$

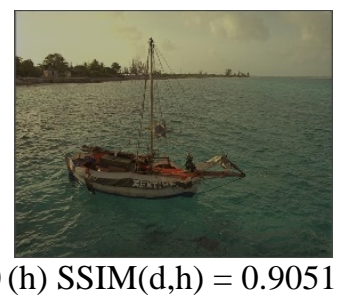

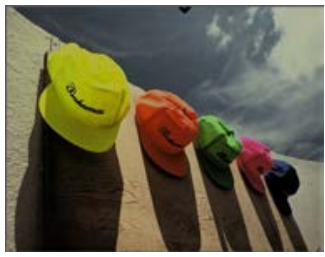

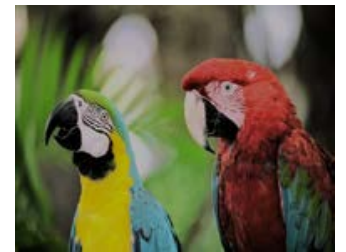

(k) $\operatorname{SSIM}(\mathrm{c}, \mathrm{k})=0.8257$ (l) $\operatorname{SSIM}(\mathrm{d}, \mathrm{l})=0.8041$
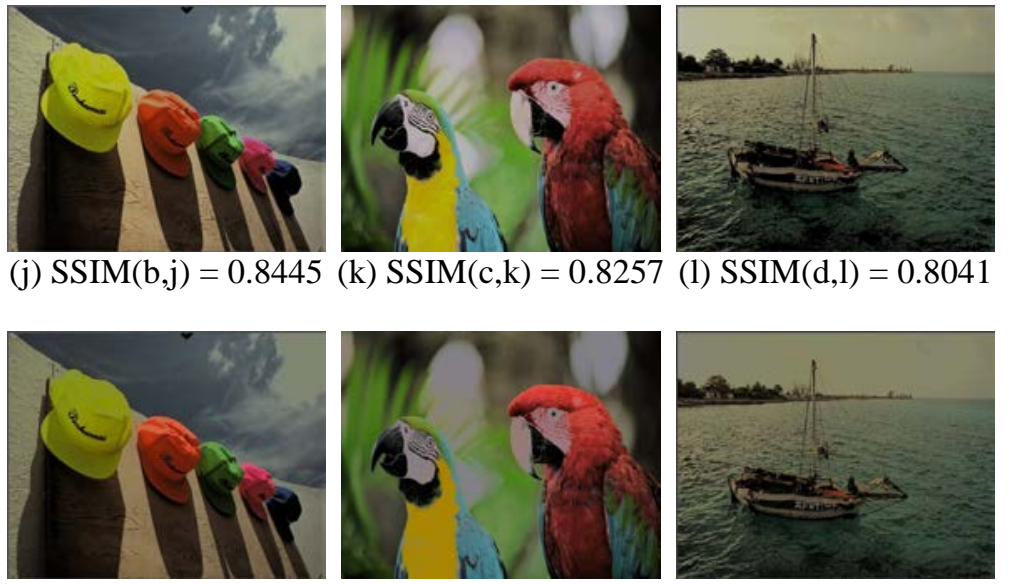

$(\mathrm{m}) \operatorname{SSIM}(\mathrm{a}, \mathrm{m})=0.9067(\mathrm{n}) \operatorname{SSIM}(\mathrm{b}, \mathrm{n})=0.8387(\mathrm{o}) \operatorname{SSIM}(\mathrm{c}, \mathrm{o})=0.8076(\mathrm{p}) \operatorname{SSIM}(\mathrm{d}, \mathrm{p})=0.8257$
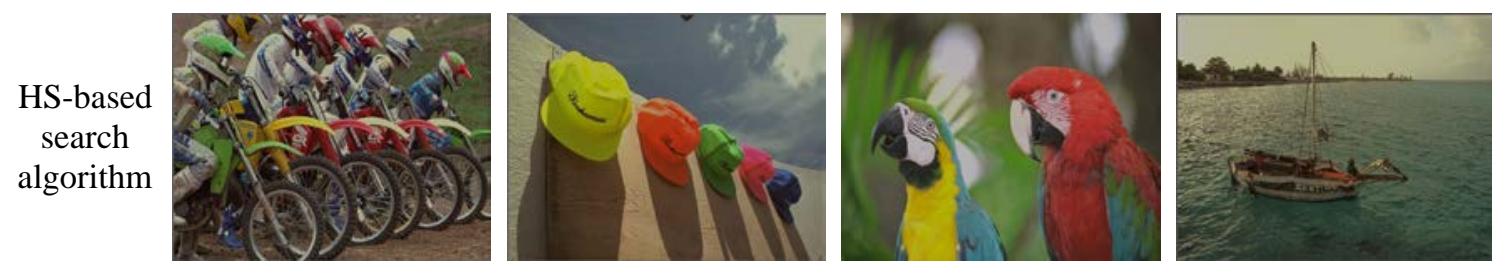

(q) $\operatorname{SSIM}(a, q)=0.9837$

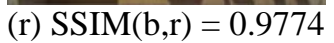

(s) $\operatorname{SSIM}(\mathrm{c}, \mathrm{s})=0.9657$

(t) $\operatorname{SSIM}(d, t)=0.9341$
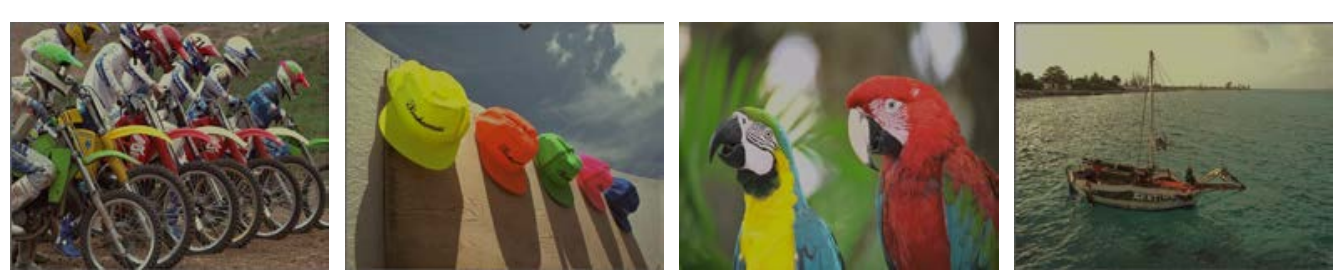

(u) $\operatorname{SSIM}(\mathrm{a}, \mathrm{u})=0.9830(\mathrm{v}) \operatorname{SSIM}(\mathrm{b}, \mathrm{v})=0.9770(\mathrm{w}) \operatorname{SSIM}(\mathrm{c}, \mathrm{w})=0.9550(\mathrm{x}) \operatorname{SSIM}(\mathrm{d}, \mathrm{x})=0.9305$

Fig. 11. Experimental results for (e)-(h) in Fig. 9 with the backlight dimmed to 0.7 times the maximum brightness. 
Table 1. SSIM indices for different dimming ratios.

\begin{tabular}{|c|c|c|c|c|c|}
\hline $\begin{array}{l}\text { Dimming } \\
\text { ratio }\end{array}$ & $\begin{array}{c}\text { Dimmed } \\
\text { result }\end{array}$ & HEBS & BCCE & $\begin{array}{c}\text { HS-based search } \\
\text { algorithm }\end{array}$ & HSPCA \\
\hline$\times 1.0$ & 1 & 0.8260 & 0.9313 & 1 & 1 \\
\hline$\times 0.9$ & 0.9905 & 0.8453 & 0.9167 & 0.9967 & 0.9960 \\
\hline$\times 0.8$ & 0.9597 & 0.8493 & 0.8892 & 0.9845 & 0.9825 \\
\hline$\times 0.7$ & 0.9031 & 0.8277 & 0.8504 & 0.9589 & 0.9549 \\
\hline
\end{tabular}

\section{Conclusion}

In this paper, we proposed an HS-based image-quality compensation technique that can be used in combination with backlight dimming to achieve both HVS-aware image quality and a high degree of power saving for TFT-LCDs. First, we combined pixel compensation and HS, and we then proposed a search algorithm that generates the optimum resultant image in terms of HVS awareness using the SSIM index. The experimental results showed that the resultant images of the HS-based search algorithm display significantly improved visual satisfaction compared to the dimmed images, and the proposed algorithm exhibits a visual performance that is superior to that of an existing HE-based technique. However, the HS-based search algorithm has the disadvantage of requiring a lengthy computation time owing to recursive processes. Thus, we developed the HSPCA to obtain the optimum SSIM-based visual quality rapidly. Based on our results, this method is approximately 28.6 times faster in terms of the computation time, while its visual satisfaction is very similar to that of the HS-based search algorithm. As future work, we plan to utilize the HSPCA in a runtime implementation.

\section{References}

[1] A. Carroll and H. Gernot, “An analysis of power consumption in a smartphone," in Proc. of USENIX annual technical conference, vol. 14, June 23-25, 2010.

https://www.usenix.org/legacy/event/usenix10/tech/full_papers/Carroll.pdf

[2] S. Kang, “SSIM preservation-based backlight dimming,” Journal of Display Technology, vol. 10, pp.247-250, January, 2014. Article (CrossRef Link)

[3] J. Chen, B. Ma, R. Li, T. Xia, and H. Cao. "Image dimming perceptual model based pixel compensation and backlight adjustment," Journal of Display Technology, Vol. 11, no. 9, pp. 744-752. 2015. Article (CrossRef Link)

[4] Z. Wang, A.C.Bovik, H.R.Sheikh and E.P.Simoncelli, "Image quality assessment: from error visibility to structural similarity,” IEEE Trans. on Image Processing, vol. 13, no. 4, pp.600-612, April, 2004. Article (CrossRef Link)

[5] C. Jung and Z. Xia, "Perceptual backlight scaling for low power liquid crystal displays based on visual saliency," in Proc. of the IEEE International Conference on Image Processing (ICIP), September 27-30, 2015. Article (CrossRef Link)

[6] S. Kang and Y. Kim, "Segmentation-based clipped error control algorithm for global backlight dimming,” Journal of Display Technology, vol 10, pp.568-573, July, 2014. Article (CrossRef Link)

[7] Y. Li, P. Chu, J. Liu, and S. Du, “A novel partitioned light guide backlight LCD for mobile devices and local dimming method with nonuniform backlight compensation," Journal of Display Technology, Vol. 10, no. 4, pp. 321-328. 2014. Article (CrossRef Link) 
[8] C. Shen, Z. Lu, Y. Hung and S. Pei, "Visual enhancement using sparsity-based image decomposition for low backlight displays," in Proc. of 2016 IEEE International Symposium Circuits and Systems (ISCAS), August, 2016. Article (CrossRef Link)

[9] J. Kim, K, Lee, J. Bae, J. Kim, and H. Kim, "Measurement of contrast reduction and its application to LCD backlight control," in Proc. of 2015 IEEE 4th Global Conference on Consumer Electronics (GCCE), pp. 294-297, 2015. Article (CrossRef Link)

[10] R. Gonzalez and R. E. Woods, Digital Image Processing, $3^{\text {rd }}$ edition, Pearson, 2007.

[11] A. Iranli, H. Fatemi and M, Pedram, "HEBS: histogram equalization for backlight scaling," in Proc. of Design, Automation and Test in Europe, pp 346-351, March 7-11, 2005. Article (CrossRef Link)

[12] W. C. Cheng and M. Pedram, "Power minimization in a backlit TFT-LCD by concurrent brightness and contrast scaling," in Proc. of the conference on Design, automation and test in Europe, vol. 1, Feb 16-20, 2004. http://dl.acm.org/citation.cfm?id=969038

[13] C. Lee, J. Kim, C. Lee and C. Kim, "Optimized brightness compensation and contrast enhancement for transmissive liquid crystal displays," IEEE Trans. on Image Processing on Circuits and Systems for Video Technology, Vol. 24, no. 4, pp. 576-590, April, 2014. Article (CrossRef Link)

[14] C. Jung, and L. Wang, "Power-constrained backlight scaling using brightness compensated contrast-tone mapping operation,” Visual Communications and Image Processing (VCIP), pp. 1-4, 2015. Article (CrossRef Link)

[15] Y. Kim, “Contrast enhancement using brightness preserving bi-histogram equalization,” IEEE Trans. Consumer Electronics, vol. 43, pp.1-8, February, 1997. Article (CrossRef Link)

[16] Y. Q. Wang, B. Chen and M. Zhang, "Image enhancement based on equal area dualistic sub-image histogram equalization method,” IEEE Trans. on Consumer Electronics, vol. 45, pp. 68-75, February, 1999. Article (CrossRef Link)

[17] T. Arici and S. Dikbas, "A histogram modification framework and its application for image contrast enhancement,” IEEE Trans.on Image Processing, vol.18, pp.1921-1935, September, 2009. Article (CrossRef Link)

[18] H. R. Sheikh, Z. Wang, and A. C. Bovik, "A statistical evaluation of recent full reference image quality assessment algorithms,” IEEE Trans. on Image Processing, vol. 15, no. 11, pp. 3440-3451, November, 2006. Article (CrossRef Link)

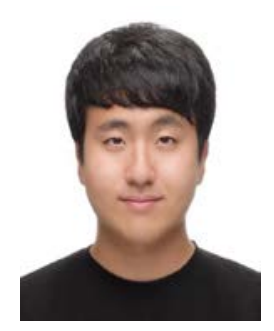

Jeong-Chan Jin received the B.S. degree in Department of Electrical and Computer Engineering from Ajou University, Suwon, Korea, in 2016. He is currently on the M.S. course in the embedded computing and systems lab (ECSL) in the same department. His research interests include image processing and parallel computing.

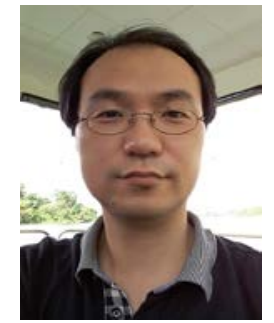

Young-Jin Kim received the B.S. and M.S. degrees in electrical engineering and the Ph.D. degree in computer science and engineering from Seoul National University, Seoul, Korea, in 1997, 1999, and 2008, respectively. From 1999 to 2003, he was with the Electronics and Telecommunications Research Institute (ETRI), Daejoen, Korea. He was an assistant professor in the Department of Computer Science and Engineering, SunMoon University, Asan, Korea from 2008 to 2011. He is currently an associate professor in the Department of Electrical and Computer Engineering, Ajou University, Suwon, Korea. His research interests include embedded systems and software, display systems and image processing, low-power technology, storage systems. 